\title{
Transseptal balloon atrial septostomy for decompression of the left atrium during extracorporeal membrane oxygenation support as a "bridge to transplantation" in dilated cardiomyopathy
}

\author{
Paweł Litwiński ${ }^{1}$, Marcin Demkow ${ }^{2}$, Małgorzata Sobieszczańska $^{3}$, Jarosław Szymański ${ }^{1}$, Józef Stolarek ${ }^{1}$, \\ Mariusz Kuśmierczyk ${ }^{1}$ \\ ${ }^{1}$ Department of Cardiosurgery and Transplantology, Institute of Cardiology, Warsaw, Poland \\ ${ }^{2}$ Department of Coronary and Structural Heart Diseases, Institute of Cardiology, Warsaw, Poland \\ ${ }^{3}$ Department of Heart Failure and Transplantology, Institute of Cardiology, Warsaw, Poland
}

Adv Interv Cardiol 2017; 13, 1 (47): 72-74

DOI: https://doi.org/10.5114/aic.2017.66190

\section{Introduction}

We report the case of a 54-year-old woman with dilated cardiomyopathy who developed severe biventricular failure unresponsive to conventional therapy. She was placed on extracorporeal membrane oxygenation (ECMO) as a bridge to decision and required transseptal balloon atrial septostomy for decompression of the left atrium during mechanical circulatory support.

\section{Case report}

A 54-year-old woman with negative family history for heart diseases was transferred to our institution with the diagnosis of dilated cardiomyopathy resulting in advanced biventricular failure. She required 3 hospitalizations during the past 6 months due to heart failure exacerbation for intravenous diuretics and inotropic therapy fulfilling the criteria of Frequent Flyer - a modifier of the INTERMACS Patient Profiles designated for a patient requiring frequent emergency visits or hospitalizations for intravenous diuretics, ultrafiltration, or brief inotropic support. The need for a heart transplant was introduced and a formal workup began. The physical examination revealed significant cardiac enlargement and findings of congestive heart failure: peripheral edema, pulsatile nontender liver edge palpable $8 \mathrm{~cm}$ below the costal margin, ascites, grossly pulsatile and distended jugular veins and yellow pigmentation of sclerae. Results of laboratory tests were as follows: bilirubin $68 \mathrm{mmol} / \mathrm{l}$, estimated glomerular filtration rate (eGFR) $44 \mathrm{ml} / \mathrm{min} / 1.73 \mathrm{~m}^{2}$,
NT-pro-BNP 8500 pg/ml, Hb 10.7 g/dl. Chest X-rays showed a grossly dilated cardiac shadow and pulmonary congestion. Echocardiography revealed a left ventricular diastolic diameter of $88 \mathrm{~mm}$ with diffuse reduction in wall motion and an ejection fraction of $10 \%$. The right ventricle was also significantly enlarged (right ventricular outflow tract (RVOT) $52 \mathrm{~mm}$ ) with hypokinesis (tricuspid annular plane systolic excursion (TAPSE) $13 \mathrm{~mm}$ ). Severe mitral and tricuspid regurgitation was also present. Coronary arteriography showed normal vessels and the cardiac catheterization revealed elevated left-ventricular end-diastolic, left atrial and pulmonary artery wedge pressures. A degree of pulmonary arterial hypertension was also present (pulmonary artery pressure (PAP) 50/27/36 mm $\mathrm{Hg}$, pulmonary artery resistance (PAR) 2.8 Wood units). Following a few days of clinical improvement she developed severe biventricular failure that was unresponsive to escalating doses of inotropes, vasodilators and diuretics. Considering the clinical profile (cardiogenic shock) and rapid deterioration, the decision was made to place the patient on short-term circulatory support as a "bridge to decision". An ECMO device was implanted with an arterial cannula inserted in the ascending aorta through a median sternotomy and the venous cannula introduced in the femoral vein. A sternotomy surgical approach was selected in the expectation of potential upgrade to other forms of mechanical circulatory support. The larger diameter cannulae used for central ECMO cannulation also allow higher blood flow rates due to reduced resistance and adequate venous drain-

\section{Corresponding author:}

Paweł Litwiński MD, PhD, Department of Cardiosurgery and Transplantology, Institute of Cardiology, 42 Alpejska St, 04-628 Warsaw-Anin, Poland, phone: +48608165 808, e-mail: open4cor@gmail.com

Received: 12.11.2016, accepted: 8.02.2017. 
age - the key factor to prevent excessive preload and severe left ventricular distension. The other advantage of central ECMO is the antegrade flow of well-oxygenated blood to the arch vessels, coronaries and the rest of the body - a crucial element if the patient also has respiratory insufficiency and severe impairment of left ventricular systolic function. Anticoagulation with unfractionated heparin for an activated partial thromboplastin time (APTT) of $>1.5$ times the normal was started a few hours after ECMO institution. The perfusion flow was maintained within $50-60 \mathrm{ml} / \mathrm{kg} / \mathrm{min}$. Following a 2-day period of stability on ECMO support the arterial blood pressure wave had nearly disappeared and the patient began to develop pulmonary edema despite pharmacologic afterload reduction, inotropic support of the failing heart, optimization of ECMO flow rates and avoidance of fluid overload. Radiographically, there was increasing pulmonary congestion progressing to a "white lung" a pressing reason for left-side venting to achieve ventricular unloading. A bedside echocardiogram demonstrated a completely akinetic and distended left ventricle with subsequent lack of aortic valve opening. We decided to perform a balloon atrial septostomy (Figure 1). The patient was transferred to the hybrid catheterization laboratory and the procedure was performed under fluoroscopic guidance. A femoral venous approach was used. Transseptal puncture was required. It was subsequently dilated using gradually larger balloons with diameters that ranged from 10 to $20 \mathrm{~mm}$. Adequacy of the atrial septal defect with left-to-right shunting was confirmed by echocardiography and assessed by improved ECMO output and resolution of pulmonary edema. There were no complications related to the procedure. The patient was transferred to the intensive care unit. Complications in the following days included surgical re-exploration due to left pleural hematoma after pleurocentesis, obturative atelectasis, bleeding from the peripheral cannulation site and infection. Because of the hemodynamic stability after balloon atrial septostomy and high risk of additional surgical interventions the patient was not placed on another ventricular assist device (VAD) and successfully bridged to transplantation after ECMO support of 63 days. Nonetheless, she required huge blood product transfusions ( 25 units of red blood cells, 32 units of fresh frozen plasma and 2 units of fresh frozen platelets). The first week after the procedure was marked by a positive evolution, a fast reduction of vasoactive drug doses and good function of the graft. The subsequent hospitalization was marked by graft rejection of grades $3 R$ to $1 R$ according to the International Society for Heart and Lung Transplantation (ISHLT) controlled with intravenous steroids. The patient was discharged from hospital after several weeks of physical rehabilitation and is currently alive.

\section{Discussion}

The ECMO, a portable miniaturized cardiopulmonary bypass device providing up to $10 \mathrm{l} / \mathrm{min}$ of blood flow with gas exchange, is a worldwide expanding technology for patients in severe cardiogenic shock. It can be used as a "bridge to recovery", a "bridge to bridge" until a long-term biventricular or left ventricular assist device can be implanted, or as a "bridge to transplantation" if there are no contraindications and no possible recovery. The application of ECMO as a "bridge to decision" allows support until the clinical situation justifies the implantation of a more permanent mechanical circulatory support (MCS) system. To achieve the best survival, MCS must be commenced in an appropriate and timely fashion, ideally before the onset or aggravation of end-organ failure. Analysis of the Interagency Registry for Mechanically Assisted Circulatory Support (INTERMACS) shows that the proportion of patients in cardiogenic shock (INTERMACS level 1) undergoing long-term ventricular assist device therapy decreased from $42 \%$ in 2006 to $14 \%$ in 2012 due to poor outcomes [1]. According to the literature, INTERMACS level 1 status is the greatest risk factor for mortality in MCS therapy. The recommendation is to use the device that can offer immediate hemodynamic stabilization and best serve the needs of the patient and is familiar to the team. Complex decisions about candidacy for transplantation or MCS should be made by a multidisciplinary, experienced team. The prognosis depends significantly on the time between the hemodynamic destabilization and reestablishment of adequate end-organ perfusion. A problem encountered with a frequency of $1-60 \%$ in patients on veno-arterial ECMO support is left heart hypertension,

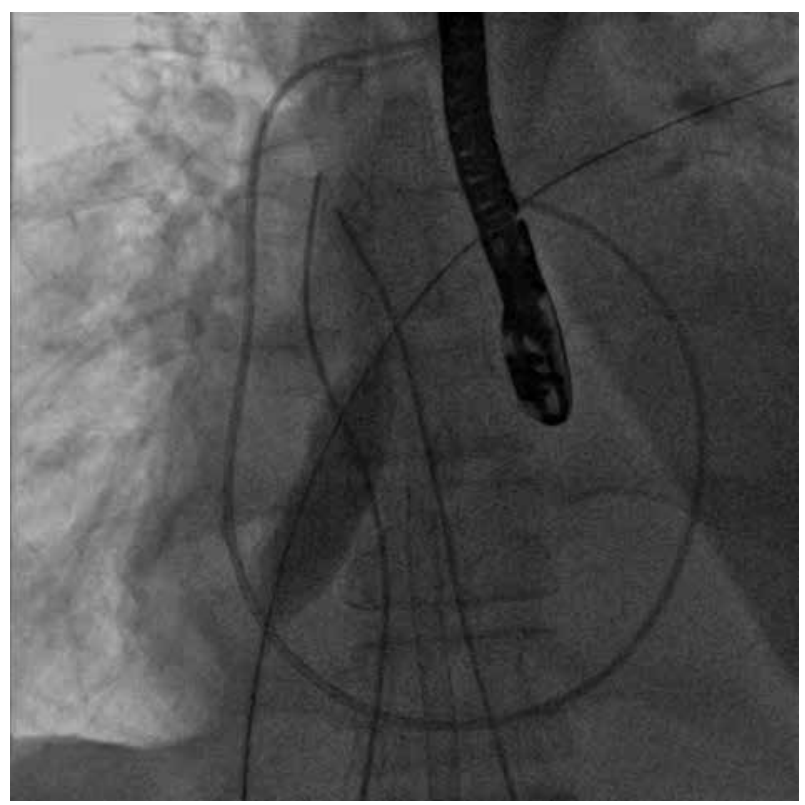

Figure 1. Transseptal balloon atrial septostomy balloon dilatation 
which may result in pulmonary edema, ventricular distension, high end-diastolic pressure, increased wall stress and subendocardial ischemia [2]. On one hand, left ventricular unloading on ECMO seems to be indicated under these circumstances. On the other hand, venting the left ventricle is invasive and can increase the likelihood of complications. Severe left ventricular distension on ECMO support may occur due to the following factors:

- Increased systemic afterload induced by veno-arterial ECMO in cases of critical left ventricular failure,

- Suboptimal venous return accompanying right heart recovery,

- Inappropriate ECMO flow rates,

- Heavy collateral bronchial flow,

- Aortic insufficiency [3].

Various methods, both surgical and nonsurgical, have been applied to decompress the left atrium or ventricle [4-8]. We describe the use of balloon dilatation of the atrial septum performed at the hybrid catheterization laboratory using transesophageal echocardiographic guidance to effectively decompress the left atrium. We strongly believe that this method can avoid pulmonary edema, but unfortunately it has no effect on left ventricular decompression in the absence of mitral insufficiency in cases of left ventricle asystole. The lack of direct left ventricular decompression can also lead to intraventricular stasis of blood with a resultant risk of thrombus formation. The advantages of our technique are the relative simplicity of the method in experienced hemodynamic hands, low risk of technique-related complications (needle perforation of the left atrium wall or pulmonary vein, pericardial effusion or tamponade), immediate hemodynamic effect, the avoidance of additional cannulae/connectors/lines, and the possibility to increase the ECMO flow and to close the septostomy percutaneously in cases of myocardial recovery. It can also be performed safely even when the patient is anticoagulated. The expected long-term patency of defects created with this technique should be sufficient. In our case the follow-up echocardiography did not demonstrate a decrease in the size of the atrial septal foramen. However, achieving unrestricted left-to-right atrial flow can be technically challenging in older patients because of the thickened septum.

\section{Conclusions}

The application of extracorporeal membrane oxygenation for circulatory support in patients with intractable heart failure has been reported. Severe left ventricular distension on ECMO assistance, a quite frequent and limiting complication of this method, may occur and result in pulmonary edema. Transseptal balloon atrial septostomy can be an effective and relatively safe method to decompress the left atrium without surgical requirement.

\section{Conflict of interest}

The authors declare no conflict of interest.

\section{References}

1. Kirklin JK, Naftel DC, Kormos RL, et al. The fourth INTERMACS annual report: 4000 implants and counting. J Heart Lung Transplant 2012; 31: 117-26.

2. Frazier EA, Faulkner SC, Seib PM, et al. Prolonged extracorporeal life support for bridging to transplant: technical and mechanical considerations. Perfusion 1997; 12: 93-8.

3. Chocron S, Perrotti A, Durst C, Aupecle B. Left ventricular venting through the right subclavian artery access during peripheral extracorporeal life support. Interact Cardiovasc Thorac Surg 2013; 17: 187-9.

4. Rupprecht L, Florchinger B, Schopka S, et al. Cardiac decompression on extracorporeal life support: a review and discussion of the literature. ASAIO J 2013; 59: 547-53.

5. Swartz M, Smith F, Byrum CJ, Alfieris GM. Transseptal catheter decompression of the left ventricule during extracorporeal membrane oxygenation. Pediatr Cardiol 2012; 33: 185-7.

6. Barbone A, Malvindi PG, Ferrara P, Tarelli G. Left ventricule unloading by percutaneous pigtail during extracorporeal membrane oxygenation. Interact Cardiovasc Thorac Surg 2011; 13: 293-5.

7. Hlavacek AM, Atz AM, Bradley SM, Bandisode VM. Left atrial decompression by percutaneous cannula placement while on extracorporeal membrane oxygenation. J Thorac Cardiovascular Surg 2005; 130: 595-6.

8. Ward KE, Tuggle DW, Gessouroun MR, et al. Transseptal decompression of the left heart during ECMO for severe myocarditis. Ann Thorac Surg 1995; 59: 749-51. 